\title{
Circular Dimensional-Permutations and Reliable Broadcasting for Hypercubes and Möbius Cubes
}

\author{
Baolei Cheng ${ }^{1,2}$, Jianxi Fan ${ }^{1, \star}$, Jiwen Yang ${ }^{1}$, and Xi Wang ${ }^{1}$ \\ 1 School of Computer Science and Technology, Soochow University, \\ Suzhou 215006, China \\ \{chengbaolei, jxfan, jwyang, 20124027002\}@suda.edu.cn \\ 2 Key Laboratory for Computer Information Processing Technology, \\ Soochow University, China
}

\begin{abstract}
Reliable broadcasting for interconnection networks can be achieved by constructing multiple independent spanning trees(ISTs) rooted at the same node. In this paper, we prove that there exists $(n-1)$ ! sets of ISTs rooted at an arbitrary node for $Q_{n}$ and $M_{n}$ based on circular dimensional-permutations of $0,1, \ldots, n-1$ and $n \geq 1$. At the same time, we give an parallel algorithm, called BCIST, which is the further study of IST problem for $Q_{n}$ and $M_{n}$ in literature. Furthermore, simulation experiments of ISTs based on JUNG framework and different sets of disjoint paths between node 1 and any node $v \in V\left(0-M_{4}\right) \backslash\{1\}$ for $0-M_{4}$ are also presented.
\end{abstract}

Keywords: dimensional-permutation, reliable broadcasting, hypercube, Möbius cube, independent spanning tree.

\section{Introduction}

It is well known that hypercubes are widely used in parallel computing systems, which have many advantageous properties such as lower node degree and diameter, higher connectivity, symmetry, and etc [21, 24]. Furthermore, by changing their links between some nodes, the variants of hypercubes, such as Möbius cubes 9], crossed cubes [20, and twisted cubes [1] were proposed, which have better properties [12, [13, [14, [16], 29].

Independent spanning trees(ISTs for short) have been used in reliable broadcasting, secure message distribution [2, reliable communication protocols 17, one-to-all broadcasting [26, the multi-node broadcasting [3, and diagnosis 6]. Therefore, the problem to construct ISTs for a given network is becoming an important issue.

However, there is a well-known conjecture on the existence of ISTs for any network [17] 31]:

^ Corresponding author.

C.-H. Hsu et al. (Eds.): NPC 2013, LNCS 8147, pp. 232-244, 2013.

(C) IFIP International Federation for Information Processing 2013 
Conjecture 1. Let $G$ be an $n$-node-connected network with $n \geq 1$. Then, there exist $n$ node-independent spanning trees rooted at any node for $G$.

In what follows, we use independent to represent node-independent. For $n \leq 4$, Conjecture 1 was solved [8], 10], [17, 31, but when $n \geq 5$, it has remained open. Consequently, researchers are interested in the study of ISTs for various special networks. Conjecture 1 has been solved for some restricted classes of networks, such as planar networks [15], product networks [23, hypercubes [25, [28, 30, Möbius cubes [5], 6], locally twisted cubes [22, crossed cubes [4, 7], twisted cubes [27, even networks [18, odd networks [19], and etc.

We say that a sequence of $n$ integers is a permutation if it contains all integers from 0 to $n-1$ exactly once. Considering the results for hypercubes and Möbius cubes, each paper in literature only considers a set of ISTs for the special network and lacks the discussion of the relation between the permutations and ISTs.

Question 1. Can all permutations of $0,1, \ldots, n-1$ be used to construct spanning tree and ISTs for the $n$-dimensional hypercube $Q_{n}$ and the $n$-dimensional Möbius cube $M_{n}$ ?

To solve this question, we adopt the definition of circular dimensionalpermutation and prove that any circular dimensional-permutation of $0,1, \ldots, n-$ 1 can be used to construct $n$ ISTs for $Q_{n}$ and $M_{n}$ in this paper, which is the further discussion of spanning trees and ISTs for $Q_{n}$ and $M_{n}$ comparing with the results in literature.

The rest of this paper is organized as follows. Section 2 presents some definitions, graph terminologies and notations. Section 3 discusses the IST problem for $Q_{n}$ and $M_{n}$ rooted at any node. We draw the conclusion of the paper in the last section.

\section{Preliminaries}

\subsection{Definition of Hypercubes, Möbius Cubes, and ISTs}

We use a unique binary string of length $n$ to denote the address of each node in the $n$-dimensional hypercube $Q_{n}$ and the $n$-dimensional Möbius cube $M_{n}$. In what follows, nodes and their addresses will be used alternatively. $Q_{n}$ is a network consists of $2^{n}$ nodes. Any two nodes of $Q_{n}$ are adjacent whenever their corresponding addresses differ in exactly one place.

$M_{n}$ is a variant of the $Q_{n}$, which has two types, 0-type $n$-dimensional Möbius cube and 1-type $n$-dimensional Möbius cube. We adopt the following definition of $M_{n}$ in [11.

Definition 1. 11] $0-M_{1}$ and 1- $M_{1}$ are both the complete graph on two nodes whose addresses are 0 and 1 . For any integer $n$ with $n \geq 2$, both $0-M_{n}$ and $1-M_{n}$ contain one 0 -type $(n-1)$-dimensional sub-Möbius cube $M_{n-1}^{0}$ and one 
1-type $(n-1)$-dimensional sub-Möbius cube $M_{n-1}^{1}$. The nodes in $M_{n-1}^{0}$ have a common prefix 0 ; the nodes in $M_{n-1}^{1}$ have a common prefix 1 . For two nodes $x=x_{n-1} x_{n-2} \ldots x_{0} \in V\left(M_{n-1}^{0}\right)$ and $y=y_{n-1} y_{n-2} \ldots y_{0} \in V\left(M_{n-1}^{1}\right)$, where $x_{n-1}=\overline{y_{n-1}}=0$,

(1) $(x, y) \in E\left(0-M_{n}\right)$ if and only if $x_{i}=y_{i}, i=0,1, \ldots, n-2$;

(2) $(x, y) \in E\left(1-M_{n}\right)$ if and only if $x_{i}=\overline{y_{i}}, i=0,1, \ldots, n-2$.

A binary string $x$ of length $n$ is denoted by $x_{n-1} x_{n-2} \ldots x_{0}$. Suppose that $u=u_{n-1} u_{n-2} \ldots u_{0}$ and $v=u_{n-1} u_{n-2} \ldots u_{l} \overline{u_{l-1}} v_{l-2} v_{l-3} \ldots v_{0}$ are two nodes in $X_{n} \in\left\{Q_{n}, M_{n}\right\}$. We say that $u$ and $v$ have a leftmost differing bit at position $l-1$. We use $\operatorname{LDF}(u, v)$ to denote the leftmost differing bit of two nodes $u$ and $v$. Given two adjacent nodes $u$ and $v$, if $\operatorname{LDF}(u, v)=d$, we say that $v$ is the $d$-neighbor of $u$ or that the edge $(u, v)$ is an edge of dimension $d$. For this purpose, let $N_{d}(u)$ denote the $d$-neighbor of $u$. We follow the definitions of path and ancestor in [7].

Two paths $P$ and $P^{\prime}$ starting from a node $u$ and ending with another node $v$ are said to be internally disjoint if $E(P) \cap E\left(P^{\prime}\right)=\varnothing$ and $V(P) \cap V\left(P^{\prime}\right)=\{u, v\}$. Two spanning trees for a network $G$ are independent if they are rooted at the same node, said $u$, and for each node $v \in V(G) \backslash\{u\}$, the two paths starting at $u$ and ending with $v$ are internally disjoint. A set of spanning trees of $G$ rooted at $v$ are called independent spanning trees if they are pairwisely independent.

\subsection{Definition of Dimensional-Permutation}

The sequence of $n$ integers is called a dimensional-permutation if it contains all integers from 0 to $n-1$ exactly one (Noting that each node in $Q_{n}$ or $M_{n}$ has $n$ neighbors, which are 0 -neighbor, 1-neighbor, ..., (n-1)-neighbor). A circular dimensional-permutation (CDP for short) is a type of permutation to put all integers from 0 to $n-1$ along a closed circle in the clockwise order [6]. Suppose that $\left\{a_{0}, a_{1}, \ldots a_{n-1}\right\}=\{0,1, \ldots, n-1\}$. All cyclic permutations of integers are equivalent in the circle,

$$
\begin{aligned}
& a_{0} \rightarrow a_{1} \rightarrow \ldots \rightarrow a_{n-1}, \\
& a_{1} \rightarrow a_{2} \rightarrow \ldots \rightarrow a_{n-1} \rightarrow a_{0}, \\
& \ldots, \\
& a_{n-1} \rightarrow a_{0} \rightarrow a_{1} \rightarrow \ldots \rightarrow a_{n-2}
\end{aligned}
$$

belong to the same CDP. The total number of CDPs is $n ! / n=(n-1)$ !. For example, the six CDPs of $0,1,2,3$ are

$$
\begin{aligned}
& 3 \rightarrow 0 \rightarrow 1 \rightarrow 2, \\
& 3 \rightarrow 0 \rightarrow 2 \rightarrow 1, \\
& 3 \rightarrow 1 \rightarrow 0 \rightarrow 2, \\
& 3 \rightarrow 1 \rightarrow 2 \rightarrow 0, \\
& 3 \rightarrow 2 \rightarrow 0 \rightarrow 1, \\
& 3 \rightarrow 2 \rightarrow 1 \rightarrow 0 .
\end{aligned}
$$




\section{An Reliable Broadcasting Algorithm Based on ISTs for Hypercubes and Möbius Cubes}

In this section, we point out that every CDP of $n$ integers $0,1,2, \ldots, n-1$ can be used to construct ISTs for $X_{n} \in\left\{Q_{n}, M_{n}\right\}$. We now present the following observation.

Observation 1. For the $n$-dimensional Möbius cube, we proved the correctness of ISTs rooted at any node based on the descending CDP $n-1, n-2, \ldots, 0[6$. In essence, the set of optimal ISTs for $Q_{n}$ in [25] can be obtained by the ascending CDP $0,1, \ldots, n-1$; the set of ISTs in [30] is similar to that in [28] for $Q_{n}$, which can be constructed by the descending CDP $n-1, n-2, \ldots, 0$. Thus, the result in this section is the further study of spanning trees and ISTs for $Q_{n}$ and $M_{n}$.

\subsection{ISTs for $Q_{n}$ and $M_{n}$ with Any Circular Dimensional-Permutation}

In what follows, we always let $u$ denote any node in $Q_{n}$ or $M_{n}$. Now we present an algorithm, called BCIST, to construct $n$ ISTs rooted at an arbitrary node $u$ for $X_{n} \in\left\{Q_{n}, M_{n}\right\}$. Fig. 1 demonstrates the construction procedures of $n$ spanning trees $T_{0}, T_{1}, \ldots, T_{n-1}$ rooted at node $u$ for $X_{n} \in\left\{Q_{n}, M_{n}\right\}$ in radial style.

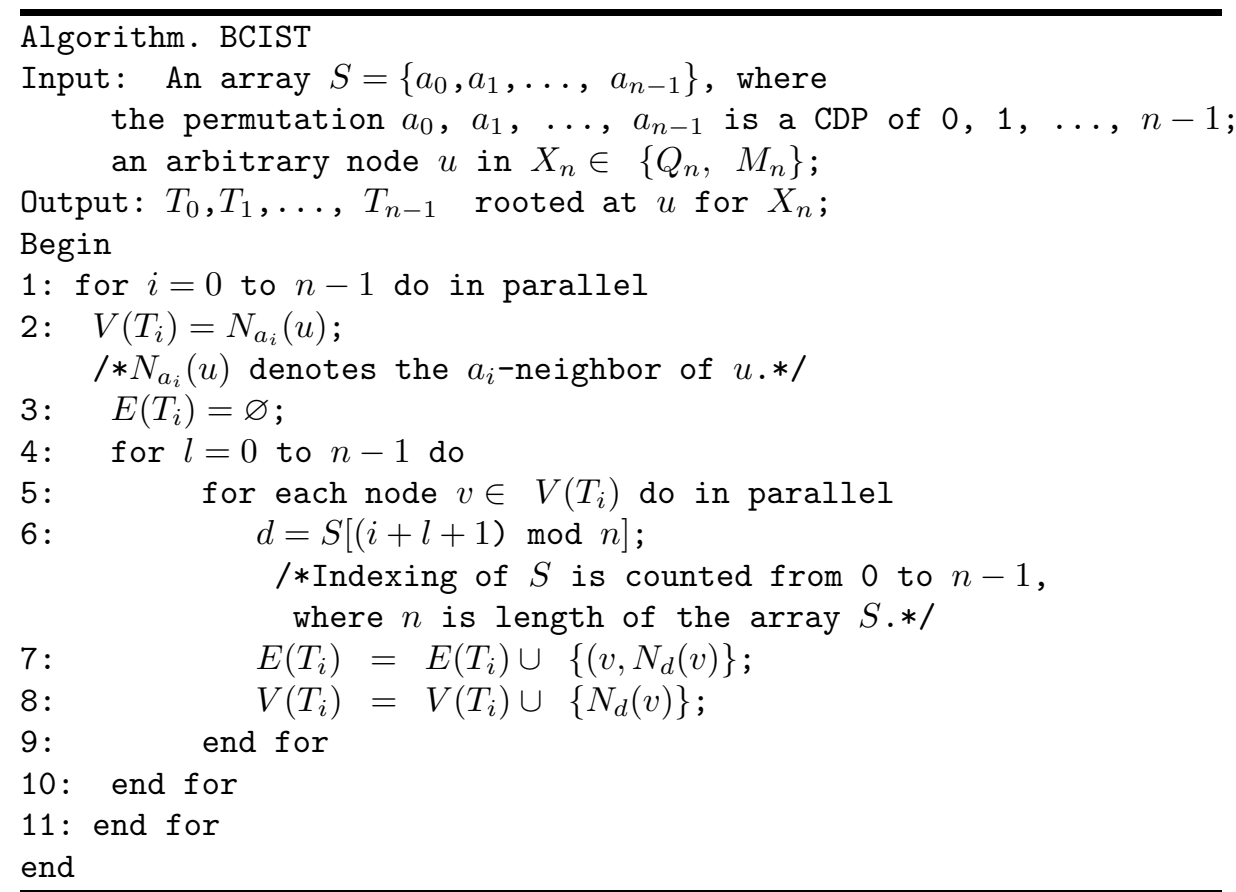




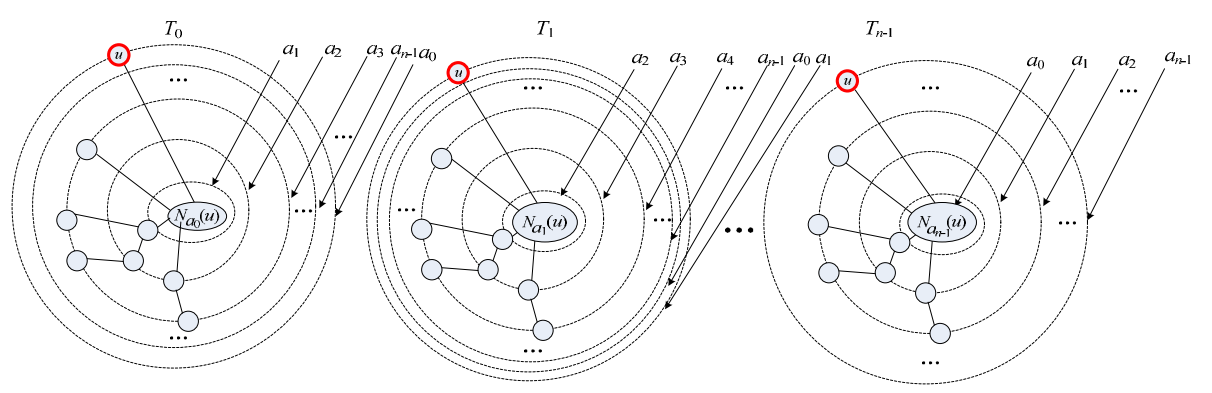

Fig. 1. Construction procedures based on algorithm BCIST

The construction procedures of $T_{0}, T_{1}, \ldots, T_{n-1}$ are similar. Take $T_{0}$ for example, the construction procedures are described as follows (See Fig. 1).

At first, there is only one node $N_{a_{0}}(u)$ in tree $T_{0}$; during the 1 st iteration $(l$ $=0)$, node $N_{a_{0}}(u)$ in $T_{0}$ is connected to its $a_{1}$-neighbor node $N_{a_{1}}\left(N_{a_{0}}(u)\right)$. Therefore, $V\left(T_{0}\right)=\left\{N_{a_{0}}(u), N_{a_{1}}\left(N_{a_{0}}(u)\right)\right\}$ and $E\left(T_{0}\right)=\left\{\left(N_{a_{0}}(u), N_{a_{1}}\left(N_{a_{0}}(u)\right)\right)\right\}$; during the 2 nd iteration $(l=1)$, each node $v$ in $T_{0}$ is connected to its $a_{2^{-}}$ neighbor node $N_{a_{2}}(v)$. Thus, the edges $\left(N_{a_{1}}\left(N_{a_{0}}(u)\right), N_{a_{2}}\left(N_{a_{1}}\left(N_{a_{0}}(u)\right)\right)\right)$ and $\left(N_{a_{0}}(u), N_{a_{2}}\left(N_{a_{0}}(u)\right)\right)$ are appended to $T_{0}$ and $V\left(T_{0}\right)=\left\{N_{a_{0}}(u), N_{a_{2}}\left(N_{a_{0}}(u)\right)\right.$, $\left.N_{a_{1}}\left(N_{a_{0}}(u)\right), N_{a_{2}}\left(N_{a_{1}}\left(N_{a_{0}}(u)\right)\right)\right\}$; during the 3rd iteration, each node $v$ in $T_{0}$ is connected to its $a_{3}$-neighbor node $N_{a_{3}}(v)$. As a result, it has doubled the number of nodes in $T_{0}$; During the $l$-th iteration, each node $v$ in $T_{0}$ is connected to its $S[l+1]$-neighbor node $N_{S[l+1]}(v)$ with $4 \leq l \leq n-2$; in the last iteration, each node $v$ in $T_{0}$ is connected to its $a_{0}$-neighbor node $N_{a_{0}}(v)$.

Consequently, $T_{0}$ is a spanning tree for $X_{n} \in\left\{Q_{n}, M_{n}\right\}$.

More examples will be shown in the next subsection. Now we give the following lemma about the relation of adjacent nodes in $M_{n}$ and $Q_{n}$.

Lemma 1. For any node $x_{n-1} x_{n-2} \ldots x_{0}$ and its $k$-neighbor node $y_{n-1} y_{n-2} \ldots y_{0}$ with $0 \leq k \leq n-1$ in $X_{n} \in\left\{M_{n}, Q_{n}\right\}$, we have $x_{n-1} x_{n-2} \ldots x_{k+1}=y_{n-1} y_{n-2} \ldots$ $y_{k+1}$ and $x_{k} \neq y_{k}$.

Based on Definition 1, the definition of $Q_{n}$, and Lemma 1, we have the following lemma.

Lemma 2. For any two nodes $x, y$ in $V\left(X_{n-1}^{x_{n-1}}\right)$, if $\operatorname{LDF}(x, y)=k$ with $0 \leq$ $k \leq n-2$ and $X_{n-1}^{x_{n-1}} \in\left\{M_{n-1}^{x_{n-1}}, Q_{n-1}^{x_{n-1}}\right\}$, then $\operatorname{LDF}\left(N_{n-1}(x), N_{n-1}(y)\right)=k$ and $N_{n-1}(x), N_{n-1}(y) \in X_{n-1}^{\overline{x_{n-1}}}$.

Lemma 3. 5] Given a walk $W: u^{(0)}, u^{(1)}=N_{m_{1}}\left(u^{(0)}\right), u^{(2)}=N_{m_{2}}\left(u^{(1)}\right), \ldots$, $u^{(k)}=N_{m_{k}}\left(u^{(k-1)}\right)$ in $M_{n}$ for any integer $k$ with $1 \leq k \leq n$, if $m_{1}, m_{2}, \ldots, m_{k}$ differ from one another and $0 \leq m_{i} \leq n-1$ for $i=1,2, \ldots, k$, then $W$ is a path.

For the convenience of proof, we define a vector $<\beta_{1}, \beta_{2}, \ldots, \beta_{n}>$ such that the set $\left\{\beta_{1}, \beta_{2}, \ldots, \beta_{n}\right\}$ equals to the set $\{0,1, \ldots, n-1\}$. 
Lemma 4. Suppose that $P$ : $u^{(0)}, u^{(1)}=N_{a_{1}}\left(u^{(0)}\right), u^{(2)}=N_{a_{2}}\left(u^{(1)}\right), \ldots$, $u^{(k)}=N_{a_{k}}\left(u^{(k-1)}\right)$ and $P^{\prime}: u^{(0)}, v^{(1)}=N_{a_{1}}\left(u^{(0)}\right), v^{(2)}=N_{a_{2^{\prime}}}\left(v^{(1)}\right), \ldots$, $v^{(t)}=N_{a_{t^{\prime}}}\left(v^{(t-1)}\right)$ are two paths in $X_{n} \in\left\{Q_{n}, M_{n}\right\}$ for any two integers $k, t$ with $1 \leq k, t \leq n$ and $a_{1} \neq a_{1}{ }^{\prime}$. If the following conditions hold:

(1) $<\beta_{i_{1}}, \beta_{i_{2}}, \ldots, \beta_{i_{k}}>=<a_{1}, a_{2}, \ldots, a_{k}>$, where $1 \leq i_{1}<i_{2}<\ldots<i_{k} \leq n$;

$(2)<\beta_{j_{1}}, \beta_{j_{2}}, \ldots, \beta_{j_{t}}>=<a_{1}{ }^{\prime}, a_{2}{ }^{\prime}, \ldots, a_{t}{ }^{\prime}>$, where $1 \leq j_{1}<j_{2}<\ldots<j_{t} \leq n$, then $V\left(\operatorname{path}\left(P, u^{(1)}, u^{(k)}\right)\right) \cap V\left(\operatorname{path}\left(P^{\prime}, v^{(1)}, v^{(t)}\right)\right)=\varnothing$.

Proof. Suppose that there exists a node $v$, such that $v \in V(P) \cap V\left(P^{\prime}\right)$. We denote $v$ as $v=u^{(i)}$ and $v=v^{(j)}$ with $1 \leq i \leq k$ and $1 \leq j \leq t$. Let $A=$ $\left\{a_{1}, a_{2}, \ldots, a_{i}\right\}$ and $B=\left\{a_{1}{ }^{\prime}, a_{2}{ }^{\prime}, \ldots, a_{j}{ }^{\prime}\right\}$. We have $M_{1}=\max ((A \cup B) \backslash(A \cap B))$. By Lemma 2, we can verify that the $M_{1}$-bit of $v$ in $P$ is different from that of $v$ in $P^{\prime}$, which is a contradiction.

Based on Lemma 4, we have the following corollary.

Corollary 1. Suppose that $P: u^{(0)}, u^{(1)}=N_{a_{1}}\left(u^{(0)}\right), u^{(2)}=N_{a_{2}}\left(u^{(1)}\right), \ldots$, $u^{(k)}=N_{a_{k}}\left(u^{(k-1)}\right)=v$ and $P^{\prime}: v^{(0)}, v^{(1)}=N_{a_{1}}\left(u^{(0)}\right), v^{(2)}=N_{a_{2}}\left(v^{(1)}\right), \ldots$, $v^{(t)}=N_{a_{t}}\left(v^{(t-1)}\right)=v$ are two paths in $X_{n} \in\left\{Q_{n}, M_{n}\right\}$ for any two integers $k, t$ with $1 \leq k, t \leq n$ and $a_{k} \neq a_{t}{ }^{\prime}$. If the following conditions hold:

(1) $<\beta_{i_{1}}, \beta_{i_{2}}, \ldots, \beta_{i_{k}}>=<a_{1}, a_{2}, \ldots, a_{k}>$, where $1 \leq i_{1}<i_{2}<\ldots<i_{k} \leq n$;

$(2)<\beta_{j_{1}}, \beta_{j_{2}}, \ldots, \beta_{j_{t}}>=<a_{1}{ }^{\prime}, a_{2}{ }^{\prime}, \ldots, a_{t}{ }^{\prime}>$, where $1 \leq j_{1}<j_{2}<\ldots<j_{t} \leq n$, then $V\left(\operatorname{path}\left(P, u^{(0)}, u^{(k)}\right)\right) \cap V\left(\operatorname{path}\left(P^{\prime}, v^{(0)}, v^{(t)}\right)\right)=\{\bar{v}\}$.

Lemma 5. Let the input $a_{0}, a_{1}, \ldots, a_{n-1}$ of algorithm BCIST be any dimensional-permutation of integers $0,1, \ldots, n-1$ and $X_{n} \in\left\{Q_{n}, M_{n}\right\} . T_{i}$ obtained by Algorithm BCIST is a spanning tree for $X_{n}$ with integer $i=$ $0,1, \ldots, n-1$.

Proof. Without loss of generality, we consider the tree $T_{0}$ obtained by algorithm BCIST. After the $n$ iterations, we have $1+2^{0}+2^{1}+\ldots+2^{n-1}=2^{n}$ nodes in tree $T_{0}$. Choosing arbitrary two nodes $v^{(1)}$ and $v^{(2)}$ from $T_{0}$, the $<N_{a_{0}}(u), v^{(1)}>-$ path can be denoted by $N_{a_{0}}(u), x^{(1)}=N_{a_{i_{1}}}\left(N_{a_{0}}(u)\right), x^{(2)}=N_{a_{i_{2}}}\left(x^{(1)}\right), \ldots$, $x^{(k)}=N_{a_{i_{k}}}\left(x^{(k-1)}\right)$ and the $<N_{a_{0}}(u), v^{(2)}>$-path can be denoted by $N_{a_{0}}(u)$, $x^{(1)}=N_{a_{j_{1}}}\left(N_{a_{0}}(u)\right), x^{(2)}=N_{a_{j_{2}}}\left(x^{(1)}\right), \ldots, x^{(m)}=N_{a_{j_{m}}}\left(x^{(m-1)}\right)$, where $0 \leq$ $i_{1}<i_{2}<\ldots<i_{k} \leq n-1$ and $0 \leq j_{1}<j_{2}<\ldots<j_{m} \leq n-1$. By algorithm BCIST, it is easy to verify $<N_{a_{0}}(u), v^{(1)}>$-path and $<N_{a_{0}}(u), v^{(2)}>$-path satisfy the conditions in Lemma 4 , which implies that $v^{(1)} \neq v^{(2)}$.

Thus, we can say that $T_{0}$ is a spanning tree rooted at $N_{a_{0}}(u)$ for $X_{n} \in$ $\left\{Q_{n}, M_{n}\right\}$. Noting that $u$ is the child of node $N_{a_{0}}(u)$ and the leaf node in $T_{0}$, that is, $T_{0}$ is a spanning tree rooted at $u$.

Lemma 6. 4 Let $T$ and $T^{\prime}$ be two spanning trees rooted at node $u$ for a network $G$. $T$ and $T^{\prime}$ are independent if and only if for every node $v \in V(G) \backslash\{u\}$, ancestor $(v, T) \cap$ ancestor $\left(v, T^{\prime}\right)=\{u\}$ and ancestor $(v, T) \cup$ ancestor $\left(v, T^{\prime}\right) \supset\{u\}$.

Lemma 7. $T_{0}, T_{1}, \ldots, T_{n-1}$ obtained by Algorithm BCIST are $n$ ISTs for $X_{n} \in$ $\left\{Q_{n}, M_{n}\right\}$. 
Proof. We have the following two cases.

Case 1. $X_{n}$ is $M_{n}$. By Lemma $5, T_{i}$ obtained by Algorithm BCIST is a spanning tree for $V\left(M_{n}\right)$ for integer $i=0,1, \ldots, n-1$. We only need to prove that $T_{i}$ and $T_{j}$ are independent for $0 \leq i \leq j \leq n-1$.

The trivial cases is $n=1$ and $n=2$. Now we consider $n$ with $n \geq 3$. The longest path $P_{1}$ in $T_{i}$ and the longest path $P_{2}$ in $T_{j}$ can be denoted by

$P_{1}: u, x_{0}=N_{a_{i}}(u), x_{1}=N_{a_{i+1}}\left(x_{0}\right), \ldots, x_{n-i-1}=N_{a_{n-1}}\left(x_{n-i-2}\right), x_{n-i}=$ $N_{a_{0}}\left(x_{n-i-1}\right), x_{n-i+1}=N_{a_{1}}\left(x_{n-i}\right), \ldots, x_{n}=N_{a_{i}}\left(x_{n-1}\right)$ and

$P_{2}: u, y_{0}=N_{a_{j}}(u), y_{1}=N_{a_{j+1}}\left(y_{0}\right), \ldots, y_{n-j-1}=N_{a_{n-1}}\left(x_{n-j-2}\right), y_{n-j}=$ $N_{a_{0}}\left(y_{n-j-1}\right), y_{n-j+1}=N_{a_{1}}\left(y_{n-j}\right), \ldots, y_{n}=N_{a_{j}}\left(y_{n-1}\right)$,

respectively, where $0 \leq i<j \leq n-1$.

Let $a_{c_{0}}=a_{i}$ and $a_{d_{0}}=a_{j}$. By lemma 6 , we only need to prove that for any $v \in V\left(T_{i}\right) \cap V\left(T_{j}\right)$, ancestor $\left(v, T_{i}\right) \cap$ ancestor $\left(v, T_{j}\right)=\{u\}$ and ancestor $\left(v, T_{i}\right)$ $\cup$ ancestor $\left(v, T_{j}\right) \supset\{u\}$. Any path in $T_{i}$ and any path in $T_{j}$ can be denoted by $P_{3}$ and $P_{4}$, respectively, as follows.

$P_{3}: u, x_{0}=N_{a_{c_{0}}=a_{i}}(u), x_{1}^{\prime}=N_{a_{c_{1}}\left(x_{i}\right)}, \ldots, x_{k}^{\prime}=N_{a_{c_{k}}}\left(x_{k-1}{ }^{\prime}\right), x_{k+1}{ }^{\prime}=$ $N_{a_{c_{0}}}\left(x_{k}{ }^{\prime}\right)$ and

$P_{4}: u, y_{0}=N_{a_{d_{0}}=a_{j}}(u), y_{1}^{\prime}=N_{a_{d_{1}}}\left(y_{i}\right), \ldots, y_{l}{ }^{\prime}=N_{a_{d_{l}}}\left(y_{l-1}{ }^{\prime}\right), y_{l+1}{ }^{\prime}=$ $N_{a_{d_{0}}}\left(y_{l}^{\prime}\right)$,

Without loss of generality, suppose that $a_{j}>a_{i}$ and $v \in V\left(P_{3}\right) \cap V\left(P_{4}\right)$. Let $v=a_{c_{w}}=a_{d_{z}}$ where $1 \leq u \leq k$ and $1 \leq z \leq m$. Based on $P_{1}, P_{2}, P_{3}$, and $P_{4}$, we define walks $W_{1}, W_{2}, W_{3}$, and $W_{4}$ as follows.

$$
\begin{aligned}
& W_{1}: a_{i}, a_{i+1}, \ldots, a_{n-1}, a_{0}, a_{1}, \ldots, a_{i}, \\
& W_{2}: a_{j}, a_{j+1}, \ldots, a_{n-1}, a_{0}, a_{1}, \ldots, a_{j}, \\
& W_{3}: a_{c_{0}}=a_{i}, a_{c_{1}}, \ldots, a_{c_{w}}, \text { and } \\
& W_{4}: a_{d_{0}}=a_{j}, a_{d_{1}}, \ldots, a_{d_{z}} .
\end{aligned}
$$

Since $a_{c_{0}} \neq a_{d_{0}}$, we have $x_{0} \neq y_{0}$, which implies that ancestor $\left(v, P_{3}\right) \cup$ ancestor $\left(v, P_{4}\right) \supset\{u\}$. We only need to prove that ancestor $\left(v, P_{3}\right) \cap$ ancestor $\left(v, P_{4}\right)=\{u\}$. We have the following Cases.

Case 1.1. $\max \left(V\left(W_{3}\right)\right) \neq \max \left(V\left(W_{4}\right)\right)$. Since $\max \left(V\left(W_{4}\right)\right) \geq a_{j}$, then we have the following subcases.

Case 1.1.1. $\max \left(V\left(W_{3}\right)\right)<a_{j}$ and $\max \left(V\left(W_{4}\right)\right)=a_{j}$. Then, each node in $V\left(P_{4}\right) \backslash\left\{y_{m+1}{ }^{\prime}\right\}$ and each node in $V\left(P_{3}\right)$ have a leftmost different bit at position $a_{j}$. Then we have $v=y_{m+1}{ }^{\prime}$ and ancestor $\left(v, P_{3}\right) \cap$ ancestor $\left(v, P_{4}\right)=\{u\}$.

Case 1.1.2. $\max \left(V\left(W_{3} \cup W_{4}\right)\right)>a_{j}$. Then, the $\max \left(V\left(W_{3} \cup W_{4}\right)\right)$-bit of $v$ in $P_{3}$ is different from that of $v$ in $P_{4}$. It is a contradiction.

Case 1.2. $\max \left(V\left(W_{3}\right)\right)=\max \left(V\left(W_{4}\right)\right)$. Then, we have the following cases.

Case 1.2.1. $\max \left(V\left(W_{3}\right)\right)=\max \left(V\left(W_{4}\right)\right)=a_{j}$. We can verify that $v \neq y_{m+1}{ }^{\prime}$ and $a_{j} \notin\left\{a_{d_{1}}, a_{d_{2}}, \ldots, a_{d_{m}}\right\}$. We can divide path ancestor $\left(v, P_{3}\right)$ into $P_{31}$ and $P_{32}$ as follows. 


$$
\begin{aligned}
& P_{31}: u, x_{0}=N_{a_{c_{0}}}(u), x_{1}^{\prime}=N_{a_{c_{1}}}\left(x_{0}\right), \ldots, x_{f-1}{ }^{\prime}=N_{a_{c_{f-1}}}\left(x_{f-2}{ }^{\prime}\right) \text { and } \\
& P_{32}: x_{f^{\prime}}=N_{a_{c_{f}}=a_{j}}\left(x_{f-1}{ }^{\prime}\right), x_{f+1}{ }^{\prime}=N_{a_{c_{f+1}}}\left(x_{f}^{\prime}\right), \ldots, x_{w^{\prime}}=N_{a_{c_{w}}}\left(x_{u-1}{ }^{\prime}\right) .
\end{aligned}
$$

By Lemma 2, each node in $V\left(P_{31}\right)$ and each node in $V\left(P_{4}\right)$ have a leftmost different bit at position $a_{j}$. Then, $V\left(P_{4}\right) \cap V\left(P_{31}\right)=\varnothing$. By Lemma $2, \operatorname{LDF}\left(x_{f}{ }^{\prime}\right.$, $\left.y_{0}\right)=\max \left(\left\{a_{c_{0}}=a_{i}, a_{c_{1}}, \ldots, a_{c(f-1)}\right\}\right)$. Let $A=\left\{a_{c_{f+1}}, a_{c_{f+2}}, \ldots, a_{c_{w}}\right\}$ and $B=$ $\left\{a_{d_{1}}, a_{d_{2}}, \ldots, a_{d_{z}}\right\}$. Furthermore, we have the following cases.

Case 1.2.1.1. $\max ((A \cup B) \backslash(A \cap B))>\operatorname{LDF}\left(x_{f^{\prime}}, y_{0}\right)$. Then, by Lemma 2 , the $\max ((A \cup B) \backslash(A \cap B))$-bit of $v$ in $P_{3}$ is different from that of $v$ in $P_{4}$, which is a contradiction.

Case 1.2.1.2. $\max ((A \cup B) \backslash(A \cap B))=\operatorname{LDF}\left(x_{f^{\prime}}, y_{0}\right)$. By Algorithm BCIST, $T_{i}$ and $T_{j}$ are constructed based on the same CDP. Then, we can verify that $a_{c_{w}} \neq a_{d_{z}}$. By Corollary 1, $V\left(P_{4}\right) \cap V\left(P_{32}\right)=\{v\}$.

Case 1.2.1.3. $\max ((A \cup B) \backslash(A \cap B))<\operatorname{LDF}\left(x_{f^{\prime}}, y_{0}\right)$. Then, by Lemma 2, the $\operatorname{LDF}\left(x_{f}{ }^{\prime}, y_{0}\right)$ bit of $v$ in $P_{3}$ is different from that of $v$ in $P_{4}$, which is a contradiction.

Case 1.2.2. $\max \left(V\left(W_{3}\right)\right)=\max \left(V\left(W_{4}\right)\right)=M_{1}>a_{j}$. We can divide path sub-path $\langle u, v\rangle$-path of $P_{3}$ into $P_{31}$ and $P_{32}$ as follows.

$$
\begin{aligned}
& P_{31}: u, x_{0}=N_{a_{c_{0}}}(u), x_{1}^{\prime}=N_{a_{c_{1}}}\left(x_{0}\right), \ldots, x_{f-1}{ }^{\prime}=N_{a_{c_{f}-1}}\left(x_{f-2}{ }^{\prime}\right) \text { and } \\
& P_{32}: x_{f}{ }^{\prime}=N_{a_{c_{f}=M_{1}}}\left(x_{f-1}{ }^{\prime}\right), x_{f+1}{ }^{\prime}=N_{a_{c_{f+1}}}\left(x_{f^{\prime}}\right), \ldots, x_{w}^{\prime}=N_{a_{c_{w}}}\left(x_{u-1}{ }^{\prime}\right) .
\end{aligned}
$$

We can divide path sub-path $\langle u, v\rangle$-path of $P_{4}$ into $P_{41}$ and $P_{42}$ as follows. $P_{41}: u, y_{j}=N_{a_{d_{0}}=a_{j}}(u), y_{1}{ }^{\prime}=N_{a_{d_{1}}}\left(y_{i}\right), \ldots, y_{h-1}{ }^{\prime}=N_{a_{d_{h-1}}}\left(y_{h-2}{ }^{\prime}\right)$, and $P_{42}: y_{h}{ }^{\prime}=N_{a_{h}}\left(y_{h-1}{ }^{\prime}\right), y_{h+1}{ }^{\prime}=N_{a_{d_{h+1}}}\left(y_{h}{ }^{\prime}\right), \ldots, y_{z}{ }^{\prime}=N_{a_{z}}\left(y_{z-1}{ }^{\prime}\right)$,

By Lemma 2, $V\left(P_{31}\right) \cap V\left(P_{41}\right)=\{u\}$. Clearly, the $M_{1}$-bit of each node in $V\left(P_{42}\right)$ is different from each node in $V\left(P_{31}\right)$, thus $V\left(P_{42}\right) \cap V\left(P_{31}\right)=\varnothing$. Similarly, we have $V\left(P_{32}\right) \cap V\left(P_{41}\right)=\varnothing$. We only need to prove that $V\left(P_{32}\right) \cap$ $V\left(P_{42}\right)=\varnothing$. Similarly to Case 1.2 .1 , we can verify that $a_{c_{w}} \neq b_{d_{z}}$. By Corollary $1, V\left(P_{32}\right) \cap V\left(P_{42}\right)=\{v\}$.

As a consequence, we have ancestor $\left(v, P_{3}\right) \cap$ ancestor $\left(v, P_{4}\right)=\{u\}$.

Case 2. $X_{n}$ is $Q_{n}$. The proof is similar to that of Case 1 .

Based on the above discussion, by Lemma 6 , the lemma holds.

Since there are $(n-1)$ ! CDPs of $0,1, \ldots, n-1$, based on Lemma 7 , we have the following theorem.

Theorem 1. Based on Algorithm BCIST, there are $(n-1)$ ! sets of ISTs for $X_{n} \in\left\{Q_{n}, M_{n}\right\}$.

Comparing with the result in 25], all the $(n-1)$ ! sets of ISTs can provide optimal reliable broadcasting for $Q_{n}$. As far as the symmetry is concerned, all the $(n-1)$ ! sets of ISTs can also provide optimal reliable broadcasting for $M_{n}$. 
(a)

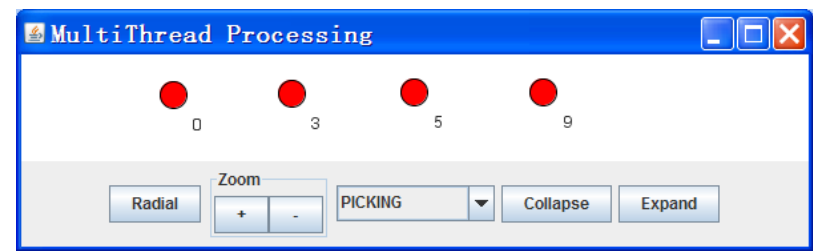

(b)
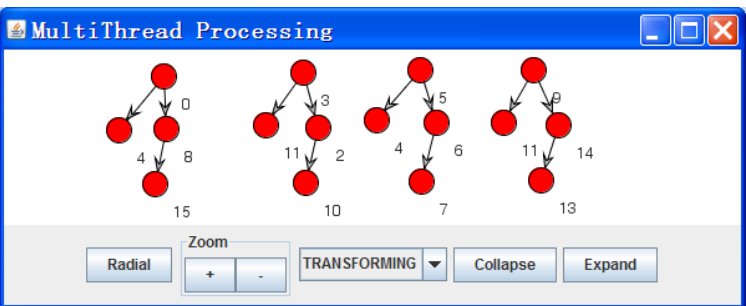

曾MultiThread Processing $\quad \square \square$

(c)

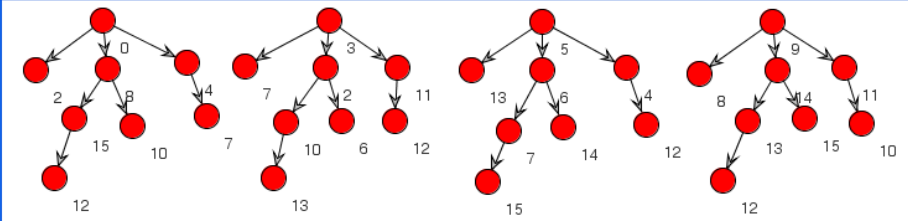

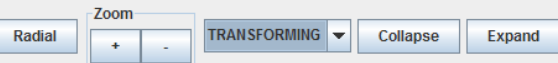

(d)

\begin{tabular}{ll}
\hline GultiThread Processing & $\square[\square$ \\
\hline
\end{tabular}

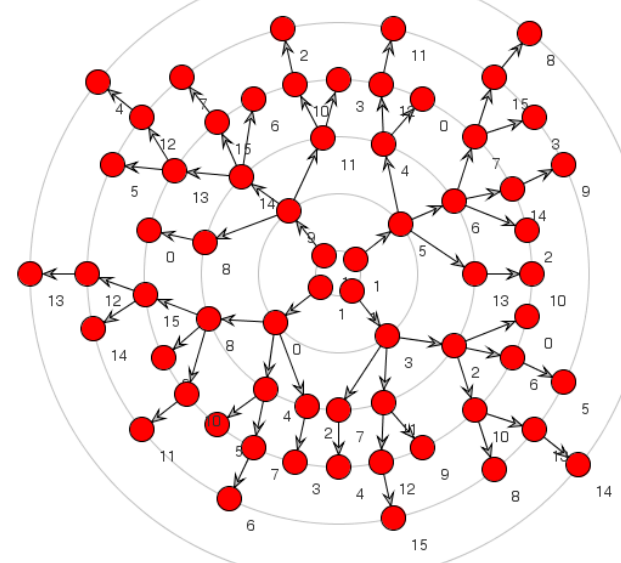

Radial

Zoom

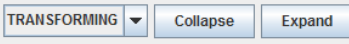

Fig. 2. Simulation experiments of ISTs based on JUNG framework. (a) Initialization; (b) The 2nd iteration; (c) The 3rd iteration; (d) The 4th iteration. 


\begin{tabular}{|c|c|c|}
\hline (a) & $V_{1}^{\downarrow}$ & 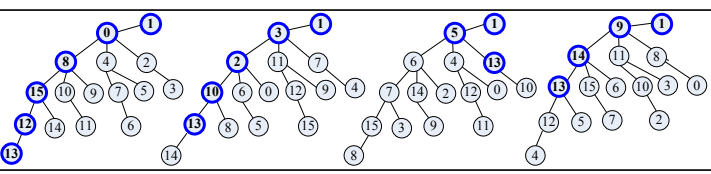 \\
\hline (b) & $\backslash_{2}^{\downarrow}$ & 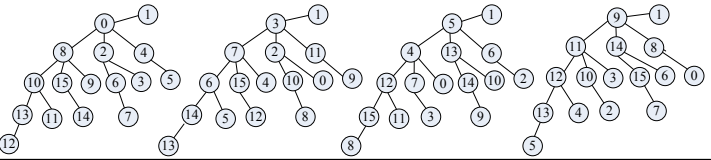 \\
\hline (c) & & 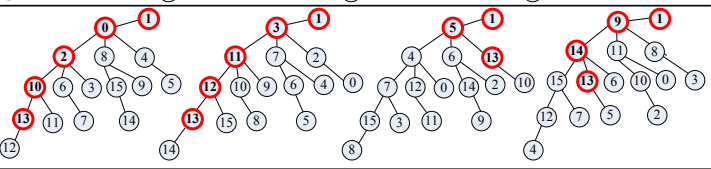 \\
\hline (d) & & 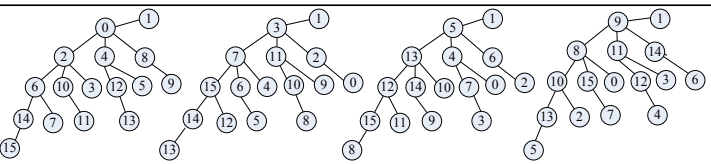 \\
\hline (e) & $V_{1}$ & 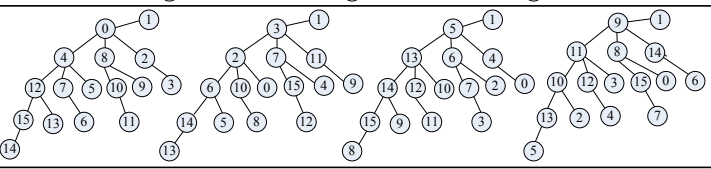 \\
\hline (f) & $\begin{array}{l}\downarrow \\
\downarrow \\
\downarrow \\
\vdots\end{array}$ & 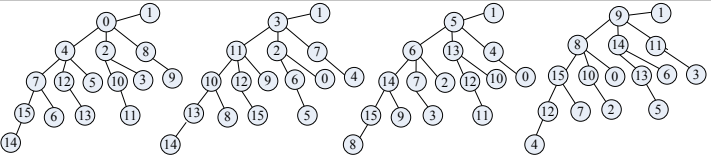 \\
\hline
\end{tabular}

Fig. 3. Example of ISTs rooted at node 1 and disjoint paths between nodes 1 and 13 in $0-M_{4}$

\subsection{Simulation Experiments of ISTs and Disjoint Paths for $0-M_{4}$}

As it is well-known that JUNG framework is a software library which provides a common and extendible language for the modeling, analysis, and visualization of data that can be represented as a network. Using Java multi-thread and JUNG framework, we can easily construct ISTs for $Q_{n}$ and $M_{n}$. For example, Fig. 2 illustrates some construction procedures of four ISTs rooted at 1 for $0-M_{4}$. The four trees can be obtained by four steps with parallel fashion.

Based on different CDPs, we can obtain multiple sets of ISTs. Thus, there are different sets of disjoint paths between arbitrary two nodes. Fig. 3)(a)-(f) show six sets of ISTs rooted at 1 for $0-M_{4}$ based on the six CDPs mentioned in Section 2.2. Clearly, there are six sets of $n$ disjoint paths between node 1 and any node $v \in V\left(0-M_{4}\right) \backslash\{1\}$, among which the total length of four disjoint paths between node 1 and node $v$ may be different. For example, the four disjoint paths between nodes 1 and 13 in Fig. 33(a) are

$$
\begin{aligned}
& 1 \rightarrow 0 \rightarrow 8 \rightarrow 15 \rightarrow 12 \rightarrow 13, \\
& 1 \rightarrow 3 \rightarrow 2 \rightarrow 10 \rightarrow 13,
\end{aligned}
$$




$$
\begin{aligned}
& 1 \rightarrow 5 \rightarrow 13, \text { and } \\
& 1 \rightarrow 9 \rightarrow 14 \rightarrow 13 .
\end{aligned}
$$

The four disjoint paths between nodes 1 and 13 in Fig. 3)(c) are

$$
\begin{aligned}
& 1 \rightarrow 0 \rightarrow 2 \rightarrow 10 \rightarrow 13, \\
& 1 \rightarrow 3 \rightarrow 11 \rightarrow 12 \rightarrow 13, \\
& 1 \rightarrow 5 \rightarrow 13, \text { and } \\
& 1 \rightarrow 9 \rightarrow 14 \rightarrow 13 .
\end{aligned}
$$

The total length of four disjoint paths between nodes 1 and 13 in Fig. 3 (c) is shorter than that in Fig. 3(a). Thus, basing on CDPs, we may easily obtain a set of $n$ disjoint paths with better performance between some pairs of nodes.

\section{Conclusions}

In this paper, we study the circular dimensional-permutations and reliable broadcasting based on ISTs for the $n$-dimensional hypercube $Q_{n}$ and the $n$-dimensional Möbius cube $M_{n}$. We prove that any circular dimensional-permutation of 0,1 , $\ldots, n-1$ can be used to construct $n$ ISTs, which is the further discussion of IST problem for $Q_{n}$ and $M_{n}$ comparing with the results in literature. Moreover, we also conduct simulation experiments of ISTs based on JUNG framework and list some disjoint paths for $0-M_{4}$.

Acknowledgment. This work is supported by National Natural Science Foundation of China (No. 61170021), Specialized Research Fund for the Doctoral Program of Higher Education (No. 20103201110018), Application Foundation Research of Suzhou of China (SYG201240), the 2011 Program for Postgraduates Research Innovation in University of Jiangsu Province (No. CXZZ11_0100), the 2012 Science and technology innovation team building program of Soochow University (SDT2012B02) and sponsored by Qing Lan Project.

\section{References}

1. Abraham, S., Padmanabhan, K.: The twisted cube topology for multiprocessors: a study in network asymmetry. J. Parallel and Distributed Computing 13(1), 104-110 (1991)

2. Bao, F., Igarashi, Y., Öhring, S.R.: Reliable broadcasting in product networks. Discrete Applied Mathematics 83(1-3), 3-20 (1998)

3. Chen, Y.-S., Chiang, C.-Y., Chen, C.-Y.: Multi-node broadcasting in all-ported 3D wormhole-routed torus using an aggregation-then-distribution strategy. J. Syst. Architect. 50(9), 575-589 (2004)

4. Cheng, B., Fan, J., Jia, X., Zhang, S.: Independent spanning trees in crossed cubes. Information Sciences 233(1), 276-289 (2013)

5. Cheng, B., Fan, J., Jia, X., Zhang, S., Chen, B.: Constructive algorithm of independent spanning trees on Möbius cubes. The Computer Journal 123 (2012), doi:10.1093/comjnl/bxs123 
6. Cheng, B., Fan, J., Jia, X., Jia, J.: Parallel construction of independent spanning trees and an application in diagnosis on Möbius Cubes. J. Supercomput. 65(3), 1279-1301 (2013)

7. Cheng, B., Fan, J., Jia, X., Wang, J.: Dimension-adjacent trees and parallel construction of independent spanning trees on crossed cubes. J. Parallel and Distributed Computing 73(5), 641-652 (2013)

8. Cheriyan, J., Maheshwari, S.N.: Finding nonseparating induced cycles and independent spanning trees in 3-connected graphs. J. Algorithms 9(4), 507-537 (1988)

9. Cull, P., Larson, S.M.: The Möbius cubes. IEEE Trans. Comput. 44(5), 647-659 (1995)

10. Curran, S., Lee, O., Yu, X.: Finding four independent trees. SIAM J. Comput. 35(5), 1023-1058 (2006)

11. Fan, J.: Diagnosability of the Möbius Cubes. IEEE Trans. Parallel Distrib. Syst. 9(9), 923-928 (1998)

12. Fan, J.: Hamilton-connectivity and cycle-embedding of the Möbius cubes. Inf. Process. Lett. 82(2), 113-117 (2002)

13. Fan, J., Jia, X.: Embedding meshes into crossed cubes. Information Sciences 177(15), 3151-3160 (2007)

14. Fan, J., Jia, X., Lin, X.: Optimal embeddings of paths with various lengths in twisted cubes. IEEE Trans. Parallel Distrib. Syst. 18(4), 511-521 (2007)

15. Huck, A.: Independent trees in planar graphs. Graphs and Combinatorics 15(1), 29-77 (1999)

16. Hsieh, S.-Y., Chen, C.-H.: Pacyclicity on Möbius cubes with maximal edge faults. Parallel Comput 30(3), 407-421 (2004)

17. Itai, A., Rodeh, M.: The multi-tree approach to reliability in distributed networks. Inform. Comput. 79(1), 43-59 (1988)

18. Kim, J.-S., Lee, H.-O., Cheng, E., Lipták, L.: Independent spanning trees on even networks. Information Sciences 181(13), 2892-2905 (2011)

19. Kim, J.-S., Lee, H.-O., Cheng, E., Lipták, L.: Optimal independent spanning trees on odd graphs. J. Supercomputing 56(2), 212-225 (2011)

20. Kulasinghe, P., Bettayeb, S.: Multiply-twisted hypercube with five or more dimensions is not vertex-transitive. Inf. Process. Lett. 53(1), 33-36 (1995)

21. Lee, S.C., Hook, L.R.: Logic and computer design in nanospace. IEEE Trans. Comput. 57(7), 965-977 (2008)

22. Liu, Y.-J., Chou, W.Y., Lan, J.K., Chen, C.: Constructing independent spanning trees for locally twisted cubes. Theoretical Computer Science 412(22), 2237-2252 (2011)

23. Obokata, K., Iwasaki, Y., Bao, F., Igarashi, Y.: Independent spanning trees of product graphs and their construction. IEICE Trans. Fundamentals of Electronics, Communications and Computer Sciences E79-A(11), 1894-1903 (1996)

24. Schlosser, M., Sintek, M., Decker, S., Nejdl, W.: HyperCuP-hypercubes, ontologies, and efficient search on peer-to-peer networks. In: Moro, G., Koubarakis, M. (eds.) AP2PC 2002. LNCS (LNAI), vol. 2530, pp. 112-124. Springer, Heidelberg (2003)

25. Tang, S.-M., Wang, Y.-L., Leu, Y.-H.: Optimal independent spanning trees on hypercubes. J. Information Science and Engineering 20(1), 143-155 (2004)

26. Tseng, Y.-C., Wang, S.-Y., Ho, C.-W.: Efficient broadcasting in wormhole-routed multicomputers: A network-partitioning approach. IEEE Trans. Parallel Distrib. Syst. 10(1), 44-61 (1999) 
27. Wang, Y., Fan, J., Zhou, G., Jia, X.: Independent spanning trees on twisted cubes. J. Parallel and Distributed Computing 72(1), 58-69 (2012)

28. Werapun, J., Intakosum, S., Boonjing, V.: An efficient parallel construction of optimal independent spanning trees on hypercubes. J. Parallel and Distributed Computing 72(12), 1713-1724 (2012)

29. Xu, J.-M., Ma, M., Lü, M.: Paths in Möbius cubes and crossed cubes. Inf. Process. Lett. 97(3), 94-97 (2006)

30. Yang, J.-S., Tang, S.-M., Chang, J.-M., Wang, Y.-L.: Parallel construction of optimal independent spanning trees on hypercubes. Parallel Comput. 33(1), 73-79 (2007)

31. Zehavi, A., Itai, A.: Three tree-paths. J. Graph Theory 13(2), 175-188 (1989) 